\title{
ASSOCIATION BETWEEN PERIODONTITIS AND METABOLIC SYNDROME IN A FAMILY HEALTH UNIT IN SALVADOR-BA
}

Julita Maria Freitas Coelho, Doutora em Saúde Coletiva, Instituto Federal de Educação da Bahia, email: julitamaria@gmail.com

Glaúcia Alencar Ponte, Mestre em Saúde Coletiva, Secretaria Municipal de Saúde de Salvador-Ba, Brasil, email: alencarglaucia@gmail.com

Isaac Suzart Gomes-Filho, Pós Doutor em Saúde Coletiva, Universidade Estadual de Feira de Santana, email: isuzart@gmail.com

Johelle de Santana Passos, Doutora em Saúde Coletiva, Universidade Federal da Bahia, email: johpassos@gmail.com

Simone Seixas da Cruz, Pós Doutora em Saúde Coletiva, Universidade Federal do Recôncavo da Bahia, email: simone.seixas1@gmail.com

Ana Claudia Morais Godoy Figueiredo, Doutora em Saúde Coletiva, Universidade de Brasília, email: aninha.morais.godoy@gmail.com

Sarah dos Santos Conceição, Doutoranda em Saúde Coletiva, Universidade de Brasília, email: sarahs.conceicao@gmail.com

Roberta Borges Silva, Mestre em Nutrição Humana, Ministério da Saúde, Brasil, email: roberta.silva.borges@gmail.com

Magno Conceição das Mêrces, Doutor em Ciências da Saúde, Universidade do Estado da Bahia, Brasil, email: mmerces@uneb.br

\begin{abstract}
Introduction: The metabolic syndrome is characterized by multiple disorders, and the in periodontitis, inflammation occurs in the tissues supporting the tooth, where in this process it is believed that the migration of oral bacteria and byproducts to the circulatory system occurs, with a systemic spread of inflammatory mediators. This study aims to verify the effect of periodontitis on the occurrence of the metabolic syndrome. Method: Cross-sectional study with 90 users of the Family Health Unit from Vale do Cambonas in Salvador-BA. Questionnaires were applied, physical / anthropometric and dental evaluation were performed, record of the results of laboratory tests was registered and evaluation of medical records. Data analysis: A

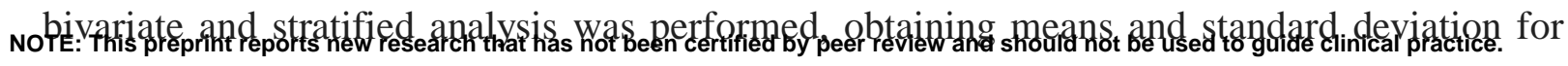
continuous variables, absolute and relative frequency for all variables, and multiple conditional
\end{abstract}


medRxiv preprint doi: https://doi.org/10.1101/2021.06.03.21258301; this version posted June 4, 2021. The copyright holder for this preprint (which was not certified by peer review) is the author/funder, who has granted medRxiv a license to display the preprint in perpetuity.

All rights reserved. No reuse allowed without permission.

logistic regression was performed to obtain the final model adjusted for potential confounders. Results: In the final sample $46,67 \%$ of participants had metabolic syndrome according NECPATP III criterion and 30, 00\% had periodontitis. From those, $20.00 \%$ had severe periodontitis, $10 \%$ moderate periodontitis and none with mild periodontitis, according to the criterion proposed by Gomes-Filho et al. (2018). There was statistical significance in the association between periodontitis and MetS (oRcrude $=2.58,95 \% \mathrm{CI}[1.02-6.55]) /($ oRadjusted $=2.63,95 \%$ CI $[1.01-6.80]$ ) and severe periodontitis and MetS (oncrude $=3.86,95 \%$ CI $[1.24-11.98]$ ) / (oRadjusted $=4.14,95 \%$ CI [1.29-13.29]). Conclusion: The main findings of this study indicate a positive association between periodontitis and metabolic syndrome, with a higher effect when the exposure was severe periodontitis.

Keywords: Metabolic Syndrome; Periodontal Disease; Periodontitis; Epidemiology.

\section{INTRODUCTION}

Metabolic syndrome (MetS) is defined as a complex disorder, represented by metabolic changes, such as dyslipidemia, arterial hypertension, glucose intolerance, central obesity and insulin resistance, which commonly occur together ${ }^{1-3}$. People diagnosed with MetS have an increased risk for cardiovascular adverse outcomes ${ }^{4}$, such as death caused by coronary disease ${ }^{5-8}$, and higher risk for type 2 diabetes ${ }^{9}$.

Thus, World Health Organization warns in regards to the increasing of noncommunicable diseases (NCD) that compose MetS and for the need of more studies in order to inform necessary control measures ${ }^{10}$. Recent studies showed the evolution of MetS, with a prevalence varying from $25 \%$ in Middle Eastern countries ${ }^{11}$ to $50.2 \%$ in India ${ }^{12}$. In the United States of America (USA), the prevalence of MetS was estimated at 32.2\% in the overall population, which increased for $34.6 \%$ in individuals older than 70 years old ${ }^{13}$.

In Brazil, in spite of the lack of studies, a high prevalence of MetS has been reported ${ }^{14}$. Specifically, on a population based study by Oliveira et al. $(2020)^{15}$, that estimated a mean prevalence of $38.4 \%$; from these, $16.7 \%$ affects people at 18 to 39 years old, $45.7 \%$ happens with those aged 40 to 59 years old and $66.1 \%$ in those aged 60 years old or above. A higher prevalence was observed on the indigenous population, $63.4 \%{ }^{16}$. Moreover, the prevalence of MetS in Brazil is higher in women, with a lower educational level and in the elderly ${ }^{15}$.

Studies show that a chronic inflammatory process may predispose MetS due to the systemic dissemination of immunologic mediators and the increase in the production of 
medRxiv preprint doi: https://doi.org/10.1101/2021.06.03.21258301; this version posted June 4, 2021. The copyright holder for this preprint (which was not certified by peer review) is the author/funder, who has granted medRxiv a license to display the preprint in perpetuity.

All rights reserved. No reuse allowed without permission.

inflammatory proteins, such as reactive $\mathrm{C}$ protein ${ }^{17-21}$. Besides, systemic inflammation can cause insulin resistance (IR) and/or hyperglycemia ${ }^{19}$.

Therefore, periodontitis acts as a precursor of systemic inflammatory processes, since there is gingival inflammation associated with damage of periodontal ligament and alveolar bone, and also with the contamination of root cement, which is associated to the presence of specific gram-negative anaerobic bacteria ${ }^{22-24}$. It is believed that there is a migration of oral bacteria and byproducts directed to the blood flow, with a systemic dissemination of local inflammatory mediators. Therefore, periodontitis may be able to stimulate ${ }^{17}$ inflammatory systemic responses and to contribute with $\mathrm{MetS}^{25-30}$.

Studies show a positive association between periodontitis and metabolic syndrome 20,26,27,31-33, varying according to population groups. Thus, the aim of the present study is to investigate the association between periodontitis and metabolic syndrome in patients of a Family Health Unit (FHU) in Vale do Cambonas, in the county of Salvador, Bahia.

\section{METHODS}

Analytical cross sectional study, with a convenience sample comprised by patients registered in Vale do Cambonas FHU. Inclusion criteria were: $\geq 30$ years old; $\geq 4$ teeth; and having registry of laboratory results of triglycerides, HDL cholesterol and fasting glucose, dated 180 days before clinical and oral examination. Up to the reporting of the present study, we included 90 participants, and analyses were obtained with a study power of $51 \%$, confidence level of $95 \%$ and exposure/non exposure ratio of 1:3, with a periodontits frequency of $40 \%$ for the non-exposed group and $20 \%$ for the exposed group ${ }^{34}$. Thus, the number of exposed participants was 27 (with periodontitis) and non-exposed was 63 (without periodontitis).

We excluded from our sample participants who have had periodontal treatment in the last three months, with systemic infections, with HIV/AIDS, and pregnant women. Data collection was performed through a questionnaire, physical/anthropometric and dental assessment, and through the analysis of laboratorial examination results and patients registries. The questionnaire comprised personal and sociodemographic data, human biology, health care and dental assistance. Physical and anthropometric examination measured blood pressure in a calm environment, after at least 5 minutes of resting, and with a previous explanation of the procedure. The participant was guided to stay seated in a comfortable position, keeping legs uncrossed and feet on the ground, leaning the back on the chair (V-DBHA, 2007). We used a stethoscope from Premium ${ }^{\circledR}$ and sphygmomanometer from P.A.MED®, both properly calibrated. 
medRxiv preprint doi: https://doi.org/10.1101/2021.06.03.21258301; this version posted June 4, 2021. The copyright holder for this preprint (which was not certified by peer review) is the author/funder, who has granted medRxiv a license to display the preprint in perpetuity.

All rights reserved. No reuse allowed without permission.

Weight and height were measured using a digital (Indi Peso Instrumentos®), with participants barefooted. Weight and height measures comprised Body Mass Index (BMI), according to Associação Brasileira para o Estudo da Obesidade $^{35}$. We measured waist circumference based on the narrowest part of the waist (the space between the lower costal border and the iliac crest), and hip circumference based on the wider part of the hip and bigger lump of the gluteal region $(150 \mathrm{~cm}-$ scale $0.5 \mathrm{~cm}$; and through the waist/hip ratio- WHR - waist circumference divided by hip circumference $)^{7,35}$.

Clinical dental evaluation was performed by a dental surgeon, previously trained, in the dental Office of FHU Vale do Cambonas. Periodontal examination was performed using a Williams probe (from Hu-Friedy®), by the measurement of probe depth, given by the distance between gingival margin and the most apical portion of probe penetration, at six sites per tooth (mesial buccal, mid-buccal, distobuccal, mesio-lingual, mid-lingual, and disto-lingual) In these sites we also verified the presence of bleeding upon probing and measured gingival recession, which comprises the distance between gingival margin and cemento-enamel junction. Dental plaque index was also evaluated for the vestibular, lingual, mesial and distal faces from dental units, and we calculated clinical attachment loss (sum between probe depth and recession measure); these procedures were both performed on the same day

Laboratory examinations (triglycerides, fasting glucose, total cholesterol and fractions) were taken by Hospital Sao Rafael (Secretaria Municipal de Saúde de Salvador). The results were obtained from patient's registries or from the examination reports held by the participants and registered in a clinical Record. Participants that were using antihypertensive or lipidlowering medication, or with a previous diabetes diagnosis also filled up information regarding blood pressure, triglycerides and fasting glucose, respectively ${ }^{7}$.

The classification by Gomes-Filho et al. $(2018)^{36}$ and Page and Eke (2007) ${ }^{37}$ and Eke $(2012)^{38}$ criterion guided the periodontitis diagnosis and its severity levels, such as severe, moderate and mild periodontitis, and the absence of periodontitis. Standardized criterion by NECP-ATP III $^{39}$ guided metabolic syndrome diagnosis, based on three or more components described in the Box below, which characterize the presence of the disease; International Diabetes Federation (IDF, 2006) ${ }^{40}$ criterion also informed its diagnosis through abdominal circumference increase plus at least two other risk factors, as described below:

Box 1: Metabolic Syndrome diagnosis criterion (NCEP-ATP III ${ }^{39}$, IDF***40 $^{40}$ ) 


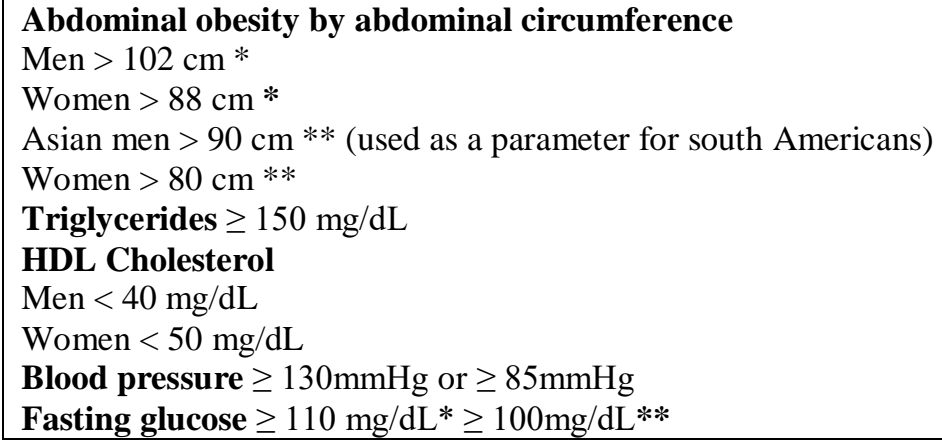

We performed a descriptive analysis for sociodemographic, lifestyle, human biology, health care and dental assistance information. Absolute and relative frequencies from relevant variables were obtained. Bivariate, stratified and multivariate analyses estimated the association between exposure and outcome variables. Pearson Chi-squared and T test estimated measures for independent variables. We adopted a $5 \%$ level of significance $(\mathrm{p}<0,05)$ and estimated Odds Ratios (OR) and its 95\% Confidence Intervals (CI 95\%).

Regarding the association between periodontitis and MetS, we evaluated the change on the effect and confounding measures, and we assumed a predictive model using MantelHaenszel test, with a significance level of $p \leq 0,20$. Empirical and theoretical basis guided the selection of potential confounding variables, considering relative differences for each covariable's adjusted measures and crude association measure above $20 \%$,

Logistic regression was performed by a multivariate analysis, with effect modifying variables verified by maximum likelihood ratio $(\mathrm{p}<0,05)$ Since we did not identify any effect change, counfounding analysis was performed by the backward strategy. We considered potential confounding variables as those that promoted a change lower than $10 \%$ on the association measure, and we obtained a final model of association between periodontitis and MetS and a final model between severe periodontitis and MetS, both adjusted by age, alcoholic beverage drinking, family income and physical activity.

The adequacy of the regression model was evaluated by the goodness fit of the model, and Hosmer-Lemeshow test was used to evaluate the goodness fit for each model. Odds Ratios (OR) were estimated for: periodontitis (Gomes-Filho et al., 2018) ${ }^{36} v s$ MetS (NECP-ATP III) ${ }^{39}$, severe periodontitis (Gomes-Filho et al., 2018) ${ }^{36}$ vs MetS (NECP-ATP III) ${ }^{39}$, periodontitis (Gomes-Filho et al., 2018) ${ }^{36}$ vs MetS (IDF) ${ }^{40}$, severe periodontitis (Gomes-Filho et al., 2018) ${ }^{36}$ vs MetS (IDF) ${ }^{40}$, periodontitis (Page e Eke, 2007; Eke et al., 2012) ${ }^{37,38}$ vs MetS (NECP-ATP III) ${ }^{39}$, severe periodontitis (Page e Eke, 2007;Eke et al., 2012) ${ }^{37,38}$ vs MetS (NECP-ATP III) ${ }^{39}$, 
medRxiv preprint doi: https://doi.org/10.1101/2021.06.03.21258301; this version posted June 4, 2021. The copyright holder for this preprint (which was not certified by peer review) is the author/funder, who has granted medRxiv a license to display the preprint in perpetuity.

All rights reserved. No reuse allowed without permission.

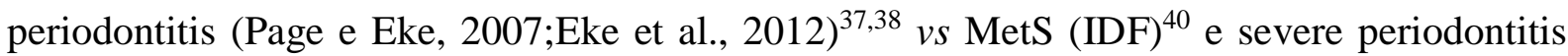

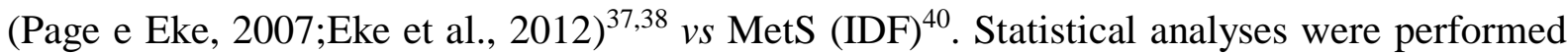
on STATA 11.

\section{RESULTS}

Ninety participants, including 57 women, comprised this study sample. Individuals diagnosed with MetS were, on average, 54 \pm 12 years old, ranging from 30 to 77 years old. There was a statistical significant difference for guidance to oral health $(p=0,02)$ regarding lifestyle and oral health, according to MetS diagnosis based on NCEP-ATP III ${ }^{39}$ criterion (TABLE 1).

Table 1 - Sociodemographic and lifestyle characteristics according to NCEP- ATP III criterion for Metabolic Syndrome diagnosis. Salvador, BA, Brazil, 2018. $(\mathrm{n}=90)$

\begin{tabular}{|c|c|c|c|c|c|}
\hline \multirow{3}{*}{ Characteristics } & \multicolumn{4}{|c|}{ MetS Diagnosis (NCEP- ATPIII) } & \multirow[b]{3}{*}{$\mathbf{p}^{*}$} \\
\hline & \multicolumn{2}{|c|}{$\begin{array}{c}\text { Yes } \\
(n=42) \\
\end{array}$} & \multicolumn{2}{|c|}{$\begin{array}{c}\text { No } \\
(n=48)\end{array}$} & \\
\hline & $\mathbf{n}$ & $\%$ & $\mathbf{n}$ & $\%$ & \\
\hline$<59$ & 22 & 52.38 & 26 & 54.17 & \multirow{5}{*}{0.87} \\
\hline$\geq 59$ & 20 & 47.62 & 22 & 45.83 & \\
\hline Mean \pm sd & \multicolumn{2}{|c|}{$54 \pm 12$} & \multicolumn{2}{|c|}{$52 \pm 11$} & \\
\hline Median & & & & & \\
\hline Mínimum-Máximum & & & & & \\
\hline \multicolumn{6}{|l|}{ Gender } \\
\hline Female & 30 & 63.64 & 27 & 47.37 & \multirow{2}{*}{0.19} \\
\hline Male & 12 & 36.36 & 21 & 52.63 & \\
\hline \multicolumn{6}{|l|}{ Race/skin color } \\
\hline Whitelyellow & 1 & 2.38 & 5 & 10.42 & \multirow{2}{*}{0.13} \\
\hline Black/Brown/other & 41 & 97.62 & 43 & 89.58 & \\
\hline \multicolumn{6}{|l|}{ Marital status } \\
\hline Married/other type & 22 & 52.38 & 24 & 50.00 & \multirow{2}{*}{0.82} \\
\hline Single/divorced/widowed & 20 & 47.62 & 24 & 50.00 & \\
\hline \multicolumn{6}{|c|}{ Educational level (years of education) } \\
\hline$>4$ years & 17 & 40.48 & 18 & 37.5 & \multirow{2}{*}{0.77} \\
\hline$\leq 4$ years & 25 & 59.52 & 30 & 62.50 & \\
\hline \multicolumn{6}{|l|}{ Family income ${ }^{1}$} \\
\hline$>1$ minimum wage & 15 & 35.71 & 23 & 47.92 & \multirow{2}{*}{0.24} \\
\hline$\leq 1$ minimum wage & 27 & 64.29 & 25 & 52.08 & \\
\hline \multicolumn{6}{|c|}{$\begin{array}{l}\text { Household density } \\
\text { (number of people per household) }\end{array}$} \\
\hline$\leq 3$ people & 28 & 66.67 & 38 & 79.17 & \multirow{2}{*}{0.18} \\
\hline$>3$ people & 14 & 33.33 & 10 & 20.83 & \\
\hline \multicolumn{6}{|l|}{ Number of children } \\
\hline$\leq 1$ child & 13 & 30.95 & 12 & 25.00 & \multirow{2}{*}{0.53} \\
\hline$\geq 2$ children & 29 & 69.05 & 36 & 75.00 & \\
\hline \multicolumn{6}{|l|}{ Current smoking habit } \\
\hline Yes & 1 & 2.38 & 5 & 10.42 & \multirow{2}{*}{0.21} \\
\hline No & 41 & 97.62 & 43 & 89.58 & \\
\hline \multicolumn{6}{|l|}{ Alcoholic beverage drinking } \\
\hline Yes & 15 & 35.71 & 14 & 29.17 & \multirow[b]{2}{*}{0.51} \\
\hline No & 27 & 64.29 & 34 & 70.83 & \\
\hline
\end{tabular}


medRxiv preprint doi: https://doi.org/10.1101/2021.06.03.21258301; this version posted June 4, 2021. The copyright holder for this preprint (which was not certified by peer review) is the author/funder, who has granted medRxiv a license to display the preprint in perpetuity.

All rights reserved. No reuse allowed without permission.

\begin{tabular}{|c|c|c|c|c|c|}
\hline \multicolumn{6}{|l|}{ Physical activity } \\
\hline Yes & 18 & 42.86 & 20 & 41.67 & \multirow{2}{*}{0.91} \\
\hline No & 24 & 57.14 & 28 & 58.33 & \\
\hline \multicolumn{6}{|c|}{ Dental surgeon consultation } \\
\hline$\geq 1$ consultation per year & 34 & 80.95 & 36 & 75.00 & \multirow{2}{*}{0.50} \\
\hline$<1$ consultation per year & 8 & 19.05 & 12 & 25.00 & \\
\hline \multicolumn{6}{|c|}{ Guided regarding oral health } \\
\hline Yes & 35 & 83.33 & 29 & 6.42 & \multirow{2}{*}{0.02} \\
\hline No & 7 & 16.67 & 19 & 39.68 & \\
\hline \multicolumn{6}{|l|}{ Tooth brushing frequency } \\
\hline$<3 x / d a y$ & 23 & 47.92 & 25 & 52.08 & \multirow{2}{*}{0.80} \\
\hline$\geq 3 x / d a y$ & 19 & 45.24 & 23 & 54.76 & \\
\hline \multicolumn{6}{|l|}{ Dental flossing } \\
\hline Yes & 21 & 50.00 & 17 & 35.42 & \multirow{2}{*}{0.16} \\
\hline No/sometimes & 21 & 50.00 & 31 & 64.58 & \\
\hline \multicolumn{6}{|l|}{ Periodontal treatment } \\
\hline Yes & 12 & 28.57 & 9 & 18.75 & \multirow{2}{*}{0.27} \\
\hline No & 30 & 71.43 & 39 & 81.25 & \\
\hline
\end{tabular}

* Statistical significance level: $\mathrm{p} \leq 0.05$.

${ }^{1}$ Minimum wage value of $\mathrm{R} \$ 954.00$ on data collection date.

According to Gomes-Filho et al. (2018) ${ }^{36}$ diagnosis criterion, 30\% of participants had periodontitis, and, from those, $40.48 \%$ had MetS; $20 \%$ had severe periodontitis, and $30.95 \%$ from those had MetS; and 10\% had moderate periodontites, $9.52 \%$ with MetS.

According to MetS diagnosis, regarding general health status and periodontitis severity, the following variables were statistically different: hypertension $(p<0,01)$, diabetes $(p=<0,01)$, BMI $(\mathrm{p}=<0,01)$, waist circumference $(\mathrm{p}=0,01)$, waist/hip ratio $(\mathrm{p}<0,01)$ and periodontitis diagnosis GOMES-FILHO et al. $(2018)^{36}(\mathrm{p}=0,04)$ (TABLE 2).

Table 2 - Distribution of characteristics related with general health status and periodontitis severity according to NCEP-ATP III diagnosis criterion for MetS. Salvador, Bahia, Brazil, 2018. $(n=90)$

\begin{tabular}{|c|c|c|c|c|c|}
\hline \multirow{2}{*}{ Characteristics } & \multicolumn{4}{|c|}{ MetS Diagnosis (NCEP- ATPIII) } & \\
\hline & \multicolumn{2}{|c|}{$\begin{array}{c}\text { Yes } \\
(n=42)\end{array}$} & \multicolumn{2}{|c|}{$\begin{array}{c}\text { No } \\
(n=48)\end{array}$} & \\
\hline Hypertension & $\mathbf{n}$ & $\%$ & $\mathbf{n}$ & $\%$ & p* \\
\hline Yes & 29 & 69.05 & 16 & 33.33 & $<0.01 *$ \\
\hline No & 13 & 30.95 & 32 & 66.67 & \\
\hline \multicolumn{6}{|l|}{ Diabetes } \\
\hline Yes & 20 & 47.62 & 4 & 8.33 & $<0.01 *$ \\
\hline No & 22 & 52.38 & 44 & 47.67 & \\
\hline
\end{tabular}


medRxiv preprint doi: https://doi.org/10.1101/2021.06.03.21258301; this version posted June 4, 2021. The copyright holder for this preprint (which was not certified by peer review) is the author/funder, who has granted medRxiv a license to display the preprint in perpetuity.

\begin{tabular}{|c|c|c|c|c|c|}
\hline \multicolumn{6}{|l|}{ Record of stroke } \\
\hline Yes & 1 & 2.38 & 1 & 2.08 & \multirow[t]{2}{*}{$0.72 *$} \\
\hline No & 41 & 97.62 & 47 & 97.92 & \\
\hline \multicolumn{6}{|l|}{ Cardiovascular Disease } \\
\hline Yes & 6 & 14.29 & 5 & 10.42 & \multirow[t]{2}{*}{0.75} \\
\hline No & 36 & 85.71 & 43 & 89.58 & \\
\hline \multicolumn{6}{|l|}{ Polycystic Ovary Syndrome (Women) $(n=57)$} \\
\hline Yes & 2 & 6.67 & 5 & 18.52 & \multirow{2}{*}{0.17} \\
\hline No & 28 & 93.33 & 22 & 81.48 & \\
\hline \multicolumn{6}{|l|}{ Kidney disease } \\
\hline Yes & 3 & 7.14 & - & 0.00 & \multirow{2}{*}{0.10} \\
\hline No & 39 & 92.86 & 48 & 100.00 & \\
\hline \multicolumn{6}{|l|}{ Hypothyroidism } \\
\hline Yes & 3 & 7.14 & 1 & 2.08 & \multirow[t]{2}{*}{0.26} \\
\hline No & 39 & 92.86 & 47 & 97.92 & \\
\hline \multicolumn{6}{|l|}{ Body Mass Index (weight/height ${ }^{2}$ ) } \\
\hline$<25$ & 3 & 7.14 & 28 & 58.33 & \multirow[t]{2}{*}{$<0.01 *$} \\
\hline$\geq 25$ & 39 & 92.86 & 20 & 41.67 & \\
\hline \multicolumn{6}{|l|}{ Waist circumference } \\
\hline$<93.24$ & 9 & 21.43 & 34 & 70.83 & \multirow[t]{2}{*}{$0.01 *$} \\
\hline$\geq 93.24$ & 33 & 78.57 & 14 & 29.17 & \\
\hline \multicolumn{6}{|l|}{ Waist/hip ratio } \\
\hline$\leq 0.87$ & 10 & 23.81 & 29 & 60.42 & \multirow{2}{*}{$<0.01 *$} \\
\hline$>0.87$ & 32 & 76.19 & 19 & 39.58 & \\
\hline \multicolumn{6}{|l|}{$\begin{array}{l}\text { Periodontitis } \\
\text { (Gomes-Filho et al., 2018) }\end{array}$} \\
\hline With periodontitis (mild, moderate, severe) & 17 & 40.48 & 10 & 20.83 & \multirow[t]{2}{*}{$0.04 *$} \\
\hline Without periodontitis & 25 & 59.52 & 38 & 79.17 & \\
\hline \multicolumn{6}{|l|}{$\begin{array}{l}\text { Periodontitis severity } \\
\text { (Gomes-Filho et al., 2018) }\end{array}$} \\
\hline Severe periodontitis & 13 & 30.95 & 5 & 10.42 & \multirow{4}{*}{$0.05^{*}$} \\
\hline Moderate periodontitis & 4 & 9.52 & 5 & 10.42 & \\
\hline Mild periodontitis & - & 0.00 & - & 0.00 & \\
\hline Without periodontitis & 25 & 59.52 & 38 & 79.17 & \\
\hline
\end{tabular}

* Statistical significance level: $\mathrm{p} \leq 0.05$.

There were statistical significant differences among participants with and without periodontitis regarding the percentual of visible plaque, probe depth, level of clinical attachment, bleeding upon probe and triglycerides, with better results for those without periodontitis (TABLE 3)

Table 3 - Clinical and laboratorial characteristics and clinical periodontal parameters from study participants, regarding presence or absence of periodontitis, according to periodontitis diagnosis criterion proposed by GomesFilho et al (2018). Salvador-Bahia, Brazil, 2018. $(n=90)$ 


\begin{tabular}{|c|c|c|c|}
\hline & $\begin{array}{l}\text { With periodontitis } \\
\qquad(n=27)\end{array}$ & $\begin{array}{c}\text { Without } \\
\text { periodontitis } \\
(n=63)\end{array}$ & \\
\hline Plaque $(\% ;$ mean \pm sd $)$ & $40.3 \pm 24.5$ & $19.5 \pm 23.6$ & \\
\hline Median & 41.6 & 11.3 & \\
\hline Minimum-Maximum & $0.0-78.9$ & $0.0-100$ & $<0.01$ \\
\hline PD (mm; mean \pm sd) & $2.5 \pm 0.75$ & $1.7 \pm 0.4$ & \\
\hline Median & 2.5 & 1.7 & \\
\hline Minimum. - Maximum & $1.8-6.0$ & $1.1-3.4$ & $<0.01$ \\
\hline LCA $(\mathbf{m m}$, mean \pm sd $)$ & $4.3 \pm 3.5$ & $2.9 \pm 0.9$ & \\
\hline Median & 4.2 & 1.9 & $<0.01$ \\
\hline Minimum - Maximum & $1.3-8.2$ & $1.2-5.4$ & \\
\hline Number of teeth $(n$, mean \pm sd) & $18.3 \pm 4.9$ & $18.1 \pm 6.1$ & \\
\hline Median & 19 & 19 & \\
\hline Minimum-Maximum & $9-27$ & $6-28$ & 0.77 \\
\hline BUP $(\%$, mean \pm sd $)$ & $48.0 \pm 18.9$ & $23.2 \pm 19.6$ & \\
\hline Median & 46.6 & 17.9 & \\
\hline Minimum-Maximum & $20.2-91.0$ & $0.0-100.0$ & $<0.01$ \\
\hline Tryglicerides (mg/dl, mean \pm sd) & $152.4 \pm 79.7$ & $138.2 \pm 80.7$ & \\
\hline Median & 157 & 117.9 & \\
\hline Minimum-Maximum & $33.7-345$ & $43.0-496$ & 0.02 \\
\hline Glucose $(\mathrm{mg} / \mathrm{dl}$, mean \pm sd) & $132.2 \pm 53.9$ & $123.9 \pm 78.0$ & \\
\hline Median & 119.0 & 95.0 & \\
\hline Minimum-Maximum & $78-258.7$ & $70-328.0$ & 0.06 \\
\hline HDL Cholesterol $(\mathrm{mg} / \mathrm{dl}$, mean \pm sd) & $50.0 \pm 14.8$ & $51.5 \pm 14.7$ & \\
\hline Median & 46.0 & 48.0 & \\
\hline Minimum - Maximum & $25-78$ & $28-97$ & 0.73 \\
\hline LDL Cholesterol (mg/dl, mean \pm sd) & $101.1 \pm 39.0$ & $119.4 \pm 30.0$ & \\
\hline Median & 91.0 & 114.0 & \\
\hline Minimum-Maximum & $27.8-232$ & $61-198.6$ & 0.23 \\
\hline BMI (mean \pm dp) & $29.2 \pm 6.0$ & $27.6 \pm 5.7$ & \\
\hline Median & 28.5 & 27.4 & \\
\hline Minimum-Maximum & $18.2-43.9$ & $16.1-46.1$ & 0.11 \\
\hline Waist circumference $(\mathrm{cm}$, mean \pm sd) & $97.1 \pm 14.0$ & $95.6 \pm 13.5$ & \\
\hline Median & 98.0 & 93.0 & \\
\hline Minimum-Maximum & $70-121$ & $61-134$ & 0.18 \\
\hline WHR (mean \pm sd) & $0.90 \pm 0.08$ & $0.88 \pm 0.8$ & \\
\hline Median & 0.90 & 0.88 & \\
\hline Minimum-Maximum & $0.73-1.1$ & $0.68-1.0$ & 0.43 \\
\hline
\end{tabular}


medRxiv preprint doi: https://doi.org/10.1101/2021.06.03.21258301; this version posted June 4, 2021. The copyright holder for this preprint (which was not certified by peer review) is the author/funder, who has granted medRxiv a license to display the preprint in perpetuity.

All rights reserved. No reuse allowed without permission.

PD: Probe depth; LCA: level of clinical attachment; BUP: bleeding upon probing; BMI: body mass index; WHR: waist/hip ratio.

* Statistical significance level: $p \leq 0,05$.

Finally, we observed a statistical significant association between periodontitis and MetS on both crude and adjusted - by age, family income, alcoholic beverage drinking and physical activity - models $\left(\mathrm{O} R_{\text {crude }}=2.58 ; \mathrm{C} 195 \%[1.02-6.55]\right) /\left(\mathrm{O} R_{\text {adjusted }}=2.63 ; \mathrm{CI} 95 \%\right.$ [1.01 - 6.80] $)$, and between severe periodontits and MetS $\left(\mathrm{O} R_{\text {crude }}=3.86 ; \mathrm{CI} 95 \%[1.24-11.98]\right) /\left(\mathrm{O} R_{\text {adjusted }}=4.14 ; \mathrm{CI} 95 \%\right.$ $[1.29$ - 13.29]) (TABLE 4).

Table 4 - Odds Ratio (OR) and Confidence Interval (CI 95\%) obtained by non conditional logistic regression for the associations between periodontitis, according to diagnosis criterion proposed by Gomes-Filho et al. (2018) and MetS, and according to NCEP-ATP III criterion, regarding periodontitis (mild, moderate and severe) vs MetS; moderate and mild periodontitis vs MetS; and severe periodontitis vs MetS. Salvador-Bahia, Brazil, 2018. $(n=90)$

\begin{tabular}{llccc}
\multicolumn{1}{c}{ PERIODONTITIS } & MODELS & OR & CI 95\% & $\mathbf{p}^{*}$ \\
\hline Peridontitis & Crude (n=90) & 2.58 & $(1.02-6.55)$ & 0.05 \\
& Adjusted $^{1}(\mathrm{n}=90)$ & 2.63 & $(1.01-6.80)$ & 0.05 \\
& Crude (n=72) & 1.17 & $(0.27-4.90)$ & 0.79 \\
$\begin{array}{l}\text { Moderate e Mild } \\
\text { Peridontitis }\end{array}$ & Adjusted $^{1}(\mathrm{n}=72)$ & 1.16 & $(0.28-4.90)$ & 0.83 \\
& Crude (n=90) $_{\text {Severe Peridontitis }}$ & 3.86 & $(1.24-11.98)$ & 0.02 \\
& Adjusted $^{1}(\mathrm{n}=90)$ & 4.14 & $(1.29-13.29)$ & 0.02 \\
\hline
\end{tabular}

${ }^{1}$ Adjusted by age, family income, alcoholic beverage drinking and physical activity.

* Statistical significance level: $\mathrm{p} \leq 0.05$.

When estimating ORs on models for the association between periodontitis ${ }^{36}$ and MetS, and regarding criterion of IDF $^{40}$ (TABLE 5), we only observed a significant statistical association between periodontitis and MetS.

Table 5 - Association between periodontitits (mild, moderate and severe) and MetS, and severe periodontitis and MetS; MetS defined by criterion from NCEP-ATP III and from IDF. Salvador-Bahia, Brazil, 2018. -

\begin{tabular}{c|cccc|ccc} 
GOMES- & \multicolumn{3}{c|}{ NCEP- ATP III } & & \multicolumn{3}{c}{ IDF } \\
\cline { 3 - 7 } $\begin{array}{c}\text { FILHO et al., } \\
2018\end{array}$ & Models & OR & CI 95\% & $\mathbf{p}^{*}$ & OR & CI 95\% & $\mathbf{p}^{*}$
\end{tabular}


medRxiv preprint doi: https://doi.org/10.1101/2021.06.03.21258301; this version posted June 4, 2021. The copyright holder for this preprint (which was not certified by peer review) is the author/funder, who has granted medRxiv a license to display the preprint in perpetuity.

All rights reserved. No reuse allowed without permission.

\begin{tabular}{cllll|llll} 
Peridontitis & Crude & 2.58 & $(1.02-6.55)$ & 0.05 & 2.78 & $(1.07-7.30)$ & 0.04 & \\
& Adjusted & $2.63^{* *}$ & $(1.01-6.80)$ & 0.05 & 2.90 & $(1.08-7.85)$ & 0.04 & \\
$\begin{array}{c}\text { Severe } \\
\text { Peridontitis }\end{array}$ & Crude & 3.86 & $(1.24-11.98)$ & 0.02 & 2.74 & $(0.89-8.51)$ & 0.08 & \\
& Adjusted & $4.14^{* * *}$ & $(1.29-13.29)$ & 0.02 & 3.08 & $(0.95-6.03)$ & 0.06 \\
\hline
\end{tabular}

* Statistical significance level: $\mathrm{p} \leq 0.05$.

** Adjusted by age, family income, alcoholic beverage drinking and physical activity

*** Adjusted by age, family income, physical activity and current smoking habit.

When these measures were estimated with periodontitis diagnosis determined by Page and Eke $(2007)^{37}$ and Eke et al. $(2012)^{38}$ criterion, and MetS diagnosis by NCEP-ATP III ${ }^{39}$ criterion and repeated for $\operatorname{IDF}^{40}$, we observed that these association only remained significant between periodontitis and MetS (determined by $\operatorname{IDF}^{40}$ criterion) $(\mathrm{p}=0.04)$, for both crude and adjusted models (TABLE 06). In order to verify the agreement level among criteria used, Kappa index was applied, which indicated a good agreement between MetS criteria (Kappa= 0.82) and moderate for periodontitis criteria (Kappa $=0.55)$.

Table 6 - Association between chronic periodontitis (mild, moderate and severe), severe periodontitis $(n=18)-$ both determined by Page and Eke (2007) and Eke et al, (2012) criterion - and MetS (by NCEP-ATP III and IDF criteria). Salvador-Bahia, Brazil, 2018. $(n=90)$

\begin{tabular}{|c|c|c|c|c|c|c|c|}
\hline \multirow{2}{*}{$\begin{array}{l}\text { PAGE\&EKE, 2007; } \\
\text { EKE et al.,2012 }\end{array}$} & \multirow{2}{*}{ Models } & \multicolumn{3}{|c|}{ NCEP-ATP III } & \multicolumn{3}{|c|}{ IDF } \\
\hline & & OR & CI $95 \%$ & $\mathbf{p}^{*}$ & OR & CI $95 \%$ & $\mathbf{p}^{*}$ \\
\hline \multirow{2}{*}{ Periodontitis } & Crude & 2.55 & $(0.96-6,70)$ & 0.06 & 2.95 & $(1.13-7.63)$ & 0.04 \\
\hline & Adjusted $^{1}$ & 2.49 & $(0.88-7.06)$ & 0.12 & 3.21 & $(1.13-9.12)$ & 0.04 \\
\hline & Crude & 2.10 & $(0.84-4.79)$ & 0.12 & 2.38 & $(0.89-5.81)$ & 0.06 \\
\hline $\begin{array}{c}\text { Severe } \\
\text { periodontitis }\end{array}$ & Adjusted $^{1}$ & 4.14 & $(0.86-5.15)$ & 0.11 & 2.40 & $(0.95-6.03)$ & 0.06 \\
\hline
\end{tabular}

${ }^{1}$ Adjusted by age, family income, current smoking habit and physical activity

* Statistical significance level: $\mathrm{p} \leq 0.05$.

\section{DISCUSSION}

Preliminary results observed in the present study indicate an association between periodontitis (Gomes-Filho et al., 2018) ${ }^{36}$ and Metabolic Syndrome (NCEP-ATP III) ${ }^{39}$, related to the presence of both periodontitis and severe periodontitis and when estimated in the model adjusted by confounding covariables: age, family income, alcoholic beverage drinking and physical activity. Studies from different countries also observed this association between MetS 
medRxiv preprint doi: https://doi.org/10.1101/2021.06.03.21258301; this version posted June 4, 2021. The copyright holder for this preprint (which was not certified by peer review) is the author/funder, who has granted medRxiv a license to display the preprint in perpetuity.

All rights reserved. No reuse allowed without permission.

and periodontitis ${ }^{20,31,32,41}$, other studies only observed it in women ${ }^{42,43}$, while one study found this association only in men ${ }^{44}$.

The bigger effect of this association regarding only severe periodontitis supports studies that only observed this association. Biological plausibility for the inter-relationship between periodontitis and MetS seems to be based by evidence showing the role of immunological markers in serum levels, such as IL-6 and TNF, that interconnect both diseases, in which combined effects have a synergic role on the coexistence of periodontitis and MetS ${ }^{19,20,26,44-48}$.

We did not observe an association between severe periodontitis, according to Gomes Filho et al.(2018) ${ }^{36}$ criterion, and MetS (IDF criterion) $)^{40}$. Despite the good agreement verified between both criteria for the diagnosis of MetS in the sample, this divergence in the finding of association of severe periodontitis reveals that the disagreements that persists in relation to the referred association, may be due to issues of this type ${ }^{47}$.

There was also a difference on the association between periodontitis, according to Page and Eke $(2007)^{37}$ and Eke et al (2012) $)^{38}$ and MetS - by both NCEP-ATP III ${ }^{40}$ and IDF diagnosis criteria $^{40}$, showing an association only for periodontitis and MetS (IDF criterion) on the adjusted model. These differences show the lack of standardizing of diagnosis criteria for periodontitis, which may be related to the existence of different evidence regarding the association of periodontitis and MetS. Furthermore, the diagnosis criterion chosen may cause bias on association measurement and compromise the quality of this measure ${ }^{49}$.

The prevalence of MetS was 46.67\%, which is similar to other Brazilian studies. Aragão, Bós and Souza $(2014)^{50}$ estimated a MetS prevalence of $55.4 \%$ in a quilombola community of Piauí state, and Borges $(2007)^{18}$, that evaluated Japanese-Brazilian participants observed a prevalence of $54.3 \%$. Studies performed in rural populations found lower estimates of MetS prevalence. Oliveira, Souza and Lima $(2006)^{51}$, in an assessment of a rural population in semiarid region of Bahia, showed a 30\% prevalence of MetS. Haab, Benvegnú and Fisher $(2012)^{52}$ found a $15.6 \%$ MetS prevalence in a rural community of Santa Rosa County in the state of Santa Catarina. These differences indicate an influence of socio-cultural and population characteristics on MetS prevalence estimates.

Regarding population assisted in health units in urban regions, the prevalence of MetS estimated in the present study is similar to the outcomes observed by Leitão and Martins $(2012)^{53}$, for instance, a $56.7 \%$ MetS prevalence in Sao Paulo regions with low socioeconomic indexes, and $34.0 \%$ in those with higher socioeconomic indexes. The higher prevalence observed in lower socioeconomic status regions points to poverty as a risk factor for MetS $\mathrm{S}^{53}$. 
medRxiv preprint doi: https://doi.org/10.1101/2021.06.03.21258301; this version posted June 4, 2021. The copyright holder for this preprint (which was not certified by peer review) is the author/funder, who has granted medRxiv a license to display the preprint in perpetuity.

All rights reserved. No reuse allowed without permission.

Metabolic Syndrome is associated with diabetes due to an increase in insulin resistance in individuals with MetS, ${ }^{9}$ which results in a higher number of cardio metabolic factors ${ }^{54}$. Thus, the results observed in a meta-analysis performed by Liu et al. (2019) ${ }^{55}$ were weak and/or inconclusive regarding the potential effect of periodontal therapy on the prevention of cardiovascular diseases, and the meta-analysis from Baeza et al. (2020) ${ }^{56}$ also indicated inconclusive evidences for the effect of periodontal therapy on glucose control.

According to Souza et al. $(2015)^{57}$, MetS and its components were significantly more common in obese individuals. The role of obesity in MetS is due to the increase of insulin resistance indexes, for instance, the gradual increase of obesity and changes in plasma metabolites, such as increases in total cholesterol and triglycerides, resulting from obesity ${ }^{58}$.

The prevalence of periodontitis observed in the present study was higher than the national average, which applyied the Community Periodontal Index (CPI) as a diagnosis criterion, where the prevalence of moderate to severe periodontitis was $15.8 \%$, and severe periodontitis only $5.8 \%$. This difference is due to different criteria used for the diagnosis of periodontitis, where the use of CPI may underestimate the disease prevalence, due to the use of índex teeth and partial examination ${ }^{60}$.

This outcome is confirmed by the elevated loss of LCA, which was significantly higher in those diagnosed with periodontitis. Similar findings were observed by Borges $(2007)^{18}$ that associated LCA loss to dental loss due to periodontal diseases. It is important to emphasize that Adachi and Kobayashi (2020) ${ }^{61}$ observed an effect independent of dental loss on MetS.

There was a positive association between periodontitis and serum triglyceride levels. Loscheet et al. (2000) ${ }^{62}$ also observed a significant increase in these levels in individuals with periodontitis. This seems to be associated to cytokine clearance in response to infection by gram-negative bacteria, where hyperlipidemia may occur due to these chemical mediators ${ }^{63}$.

This preliminary study has limitations, such as the insufficient sample size and its convenience selection method. Initially, it limits the study power, influencing on outcome significance and restricting the generalization of results. Moreover, this is a cross-sectional study, which does not allow inferences on cause-effect relationship between periodontitis and MetS, although they are interconnected.

\section{CONCLUSIONS}

The main results from the present study indicate the positive association between periodontitis and metabolic syndrome, where there was observed a higher effect when associated only to severe periodontitis cases. These results enhance the significant association 
medRxiv preprint doi: https://doi.org/10.1101/2021.06.03.21258301; this version posted June 4, 2021. The copyright holder for this preprint (which was not certified by peer review) is the author/funder, who has granted medRxiv a license to display the preprint in perpetuity.

All rights reserved. No reuse allowed without permission.

between periodontitis and MetS shown in literature, especially regarding periodontitis severity. The variety of findings and oppositions that are still present in the literature, however, show that this association is not yet well determined, showing a need for more studies that show this association. Finally, it is unquestionable the role of oral teams on individuals health, especially public, raising a professional profile more integrated with the promotion of periodontal health and overall wellbeing of health services users.

\section{REFERENCES}

1. Grundy SM, Cleeman JI, Bairey Merz CN, Brewer HB, Clark LT, Hunninghake DB, et al. Implications of recent clinical trials for the National Cholesterol Education Program Adult Treatment Panel III guidelines. Circulation. 2004;110(2):227-39.

2. Marchetti E, Monaco A, Procaccini L, Mummolo S, Gatto R, Tetè S, et al. Periodontal disease: The influence of metabolic syndrome. Nutr Metab. 2012;9:1-13.

3. Alberti KGMM, Eckel RH, Grundy SM, Zimmet PZ, Cleeman JI, Donato KA, et al. Harmonizing the metabolic syndrome: A joint interim statement of the international diabetes federation task force on epidemiology and prevention; National heart, lung, and blood institute; American heart association; World heart federation; International . Circulation. 2009;120(16):1640-5.

4. Schmidt MI, Duncan BB, E Silva GA, Menezes AM, Monteiro CA, Barreto SM, et al. Chronic non-communicable diseases in Brazil: Burden and current challenges. Lancet [Internet]. 2011;377(9781):1949-61. Available from: http://dx.doi.org/10.1016/S01406736(11)60135-9

5. Raposo L, Severo M, Barros H, Santos AC. The prevalence of the metabolic syndrome in Portugal: The PORMETS study. BMC Public Health. 2017;17(1):1-9.

6. Tobin JN, Zazula T. National Cholesterol Education Program (Ncep). Encycl Heal Behav. 2012;

7. Sociedade Brasileira de Cardiologia. Diagnóstico e tratamento. Arq Bras Cardiol [Internet]. 2005;84:1-27. Available from: http://pesquisa.bvsalud.org/bvsms/resource/pt/mis-23299

8. Zeller M, Steg G, Ravisy J, Laurent Y, Janin-Manificat P, Huiller S, et al. Prevalence of the metabolic syndrome in acute myocardial infarction and its impact on hospital outcomes. Int J Diabetes Dev Ctries. 2009;29(2):52-5.

9. Penalva DQF. Síndrome metabólica: diagnóstico e tratamento. Rev Med. 2008;87(4):245.

10. WHO. World Health Organization. World health statistics 2018: monitoring health for 
medRxiv preprint doi: https://doi.org/10.1101/2021.06.03.21258301; this version posted June 4, 2021. The copyright holder for this preprint (which was not certified by peer review) is the author/funder, who has granted medRxiv a license to display the preprint in perpetuity.

All rights reserved. No reuse allowed without permission.

the SDGs, sustainable development goals [Internet]. [cited 2019 Jan 21]. Available from: https://apps.who.int/iris/bitstream/ handle/10665/272596/9789241565585eng.pdf?ua=1

11. Ansarimoghaddam A, Adineh HA, Zareban I, Iranpour S, HosseinZadeh A, Kh F. Prevalence of metabolic syndrome in Middle-East countries: Meta-analysis of crosssectional studies. Diabetes Metab Syndr Clin Res Rev [Internet]. 2018;12(2):195-201. Available from: http://dx.doi.org/10.1016/j.dsx.2017.11.004

12. Subramani SK, Mahajan S, Chauhan P, Yadav D, Mishra M, Pakkirisamy U, et al. Prevalence of metabolic syndrome in Gwalior region of Central India: A comparative study using NCEP ATP III, IDF and Harmonized criteria. Diabetes Metab Syndr Clin Res Rev [Internet]. 2019;13(1):816-21. Available from: https://doi.org/10.1016/j.dsx.2018.12.003

13. Sumner AD, Sardi GL, Reed JF. Components of the Metabolic Syndrome Differ Between Young and Old Adults in the US Population. J Clin Hypertens. 2012;14(8):502-6.

14. Carvalho Vidigal F, Bressan J, Babio N, Salas-Salvadó J. Prevalence of metabolic syndrome in Brazilian adults: A systematic review. BMC Public Health. 2013;13(1).

15. Oliveira LVA, Dos Santos BNS, Machado ÍE, Malta DC, Velasquez-Melendez G, Felisbino-Mendes MS. Prevalence of the metabolic syndrome and its components in the Brazilian adult population. Cienc e Saude Coletiva. 2020;25(11):4269-80.

16. Rocha AKS Da, Bós ÂJG, Huttner É, Machado DC. Prevalência da síndrome metabólica em indígenas com mais de 40 anos no Rio Grande do Sul, Brasil. Rev Panam Salud Publica/Pan Am J Public Heal. 2011;29(1):41-5.

17. Scannapieco FA. Periodontal inflammation: from gingivitis to systemic disease? Compend Contin Educ Dent. 2004;25(7 Suppl 1):16-25.

18. Borges PKDO, Gimeno SGA, Tomita NE, Ferreira SR, Barros N, Cardoso MA, et al. Prevalência e características associadas à síndrome metabólica em nipo-brasileiros com e sem doença periodontal. Cad Saude Publica. 2007;23(3):657-68.

19. Chen LP, Hsu SP, Peng Y Sen, Chiang CK, Hung KY. Periodontal disease is associated with metabolic syndrome in hemodialysis patients. Nephrol Dial Transplant. 2011;26(12):4068-73.

20. Han DH, Lim SY, Sun BC, Paek D, Kim HD. The association of metabolic syndrome with periodontal disease is confounded by age and smoking in a Korean population: The Shiwha-Banwol environmental health study. J Clin Periodontol. 2010;37(7):609-16. 
medRxiv preprint doi: https://doi.org/10.1101/2021.06.03.21258301; this version posted June 4, 2021. The copyright holder for this preprint (which was not certified by peer review) is the author/funder, who has granted medRxiv a license to display the preprint in perpetuity.

All rights reserved. No reuse allowed without permission.

21. Weisberg SP, Connors T, Zhu Y, Baldwin M, Lin W-H, Wontakal S, et al. Antibody responses to SARS-CoV2 are distinct in children with MIS-C compared to adults with COVID-19. medRxiv Prepr Serv Heal Sci [Internet]. 2020; Available from: http://www.ncbi.nlm.nih.gov/pubmed/32699861\%0Ahttp://www.pubmedcentral.nih.go v/articlerender.fcgi?artid=PMC7373150

22. Srivastava M, Srivastava R, Verma P, Gautam A. Metabolic syndrome and periodontal disease: An overview for physicians Mahesh. J Fam Med Prim Care [Internet]. 2017;6(2):169-70. Available from: http://www.jfmpc.com/article.asp?issn=22494863; year $=2017$; volume $=6$; issue $=1$; spage $=169$; epage $=170 ;$ aulast $=$ Faizi

23. Haffajee AD, Socransky SS. Microbial etiological agents of destructive periodontal diseases. Periodontol 2000. 1994;5(1):78-111.

24. Linden GJ, Lyons A, Scannapieco FA. Periodontal systemic associations: Review of the evidence. J Clin Periodontol. 2013;40(SUPPL. 14):8-19.

25. Zhao J, Zhu XY, Ren Y, Li JY. Analysis of the correlation between periodontal disease and metabolic syndrome among coal mine workers: A clinical study. Medicine (Baltimore). 2020;99(33):e21566.

26. Gomes-Filho IS, Balinha I da SCE, da Cruz SS, Trindade SC, Cerqueira E de MM, Passos-Soares $\mathbf{J}$ de $\mathrm{S}$, et al. Moderate and severe periodontitis are positively associated with metabolic syndrome. Clin Oral Investig. 2020;

27. Bandiwadekar AS, Shanbhag N, Madhuniranjanswamy M, Khanagar S. Association of Periodontitis With Metabolic Syndrome: A Case-Control Study. J Int Soc Prev Community Dent. 2020;10(4):34-7.

28. Suzuki D, Yamada SI, Sakurai A, Karasawa I, Kondo E, Sakai H, et al. Correlations between the properties of saliva and metabolic syndrome A prospective observational study. Med (United States). 2020;99(51).

29. Gobin R, Tian D, Liu Q, Wang J. Periodontal diseases and the risk of metabolic syndrome: An updated systematic review and meta-analysis. Front Endocrinol (Lausanne). 2020;11(June):1035-57.

30. Teixeira FCF, Marin-Leon L, Gomes EP, Pedrão AMN, Pereira A da C, Francisco PMSB. Relationship between periodontitis and subclinical risk indicators for chronic non-communicable diseases. Braz Oral Res. 2020;34:1-10.

31. Kwon YE, Ha JE, Paik D Il, Jin BH, Bae KH. The relationship between periodontitis and metabolic syndrome among a Korean nationally representative sample of adults. J Clin Periodontol. 2011;38(9):781-6. 
medRxiv preprint doi: https://doi.org/10.1101/2021.06.03.21258301; this version posted June 4, 2021. The copyright holder for this preprint (which was not certified by peer review) is the author/funder, who has granted medRxiv a license to display the preprint in perpetuity.

32. Musskopf ML, Daudt LD, Weidlich P, Gerchman F, Gross JL, Oppermann R V. Metabolic syndrome as a risk indicator for periodontal disease and tooth loss. Clin Oral Investig [Internet]. 2017;21(2):675-83. Available from: http://dx.doi.org/10.1007/s00784-016-1935-8

33. Montero E, Molina A, Carasol M, Fernández-Meseguer A, Calvo-Bonacho E, Teresa García-Margallo M, et al. The association between metabolic syndrome and periodontitis in Spain: Results from the WORALTH (Workers' ORAL healTH) Study. J Clin Periodontol. 2021;48(1):37-49.

34. Benguigui C, Bongard V, Ruidavets JB, Chamontin B, Sixou M, Ferrières J, et al. Metabolic syndrome, insulin resistance, and periodontitis: A cross-sectional study in a middle-aged French population. J Clin Periodontol. 2010;37(7):601-8.

35. Associação Brasileira para o estudo da obesidade e da síndrome metabólica (ABESO). Diretrizes brasileiras de obesidade 2016. VI Diretrizes Bras Obesidade. 2016;7-186.

36. Gomes-Filho IS, Trindade SC, Passos-Soares J de S, Figueiredo ACMG, Vianna MIP, Hintz AM, et al. Clinical diagnosis criteria for periodontal disease: an update. J Dent Heal Oral Disord Ther. 2018;9(5):354-6.

37. Page RC, Eke PI. Case Definitions for Use in Population-Based Surveillance of Periodontitis. J Periodontol [Internet]. 2007;78(7s):1387-99. Available from: http://www.joponline.org/doi/10.1902/jop.2007.060264

38. Eke PI, Page RC, Wei L, Thornton-Evans G, Genco RJ. Update of the Case Definitions for Population-Based Surveillance of Periodontitis. J Periodontol [Internet]. 2012;83(12):1449-54.

Available from: http://www.joponline.org/doi/10.1902/jop.2012.110664

39. NCEP. National Cholesterol Education Program.Expert panel on detection, evaluation, and treatment of high blood cholesterol in adults (Adult Treatment Panel III). JAMA. 2001;285(19):2486-97.

40. IDF.International Diabetes Federation. The IDF consensus worldwide definition of metabolic syndrome. Bruxela, Bélgica, 2006. [cited 2017 Mar 16]. Available from: http://www.idf.org/

41. Kumar N, Bhardwaj A, Negi PC, Jhingta PK, Sharma D, Bhardwaj VK. Association of chronic periodontitis with metabolic syndrome: A cross-sectional study. J Indian Soc Periodontol. 2016;20(3):324-9.

42. Andriankaja OM, Sreenivasa S, Dunford R, DeNardin E. Association between metabolic syndrome and periodontal disease. Aust Dent J. 2010;55(3):252-9. 
medRxiv preprint doi: https://doi.org/10.1101/2021.06.03.21258301; this version posted June 4, 2021. The copyright holder for this preprint (which was not certified by peer review) is the author/funder, who has granted medRxiv a license to display the preprint in perpetuity.

43. Furuta M, Shimazaki Y, Takeshita T, Shibata Y, Akifusa S, Eshima N, et al. Gender differences in the association between metabolic syndrome and periodontal disease: The Hisayama Study. J Clin Periodontol. 2013;40(8):743-52.

44. Kim OS, Shin MH, Kweon SS, Lee YH, Kim OJ, Kim YJ, et al. The severity of periodontitis and metabolic syndrome in Korean population: The Dong-gu study. J Periodontal Res. 2018;53(3):362-8.

45. Sora ND, Marlow NM, Bandyopadhyay D, Leite RS, Slate EH, Fernandes JK. Metabolic syndrome and periodontitis in Gullah African Americans with type 2 diabetes mellitus. J Clin Periodontol. 2013;40(6):599-606.

46. Alhabashneh R, Khader Y, herra Z, Assad F. The association between periodontal disease and metabolic syndrome among outpatients with diabetes in Jordan. J Diabetes Metab Disord [Internet]. 2015;14(1):1-7. Available from: http://dx.doi.org/10.1186/s40200-015-0192-8

47. Gomes-Filho IS, das Mercês MC, de Santana Passos-Soares J, Seixas da Cruz S, Teixeira Ladeia AM, Trindade SC, et al. Severity of Periodontitis and Metabolic Syndrome: Is There an Association? J Periodontol. 2016;87(4):357-66.

48. Pham TAV. The association between periodontal disease severity and metabolic syndrome in Vietnamese patients. Int J Dent Hyg. 2018;16(4):484-91.

49. Gomes-Filho IS, Cruz SS, Rezende EJC, Dos Santos CAST, Soledade KR, Magalhães MA, et al. Exposure measurement in the association between periodontal disease and prematurity/low birth weight. J Clin Periodontol. 2007;34(11):957-63.

50. Aragão JA, Bós ÂJG, Souza GC. Síndrome Metabólica Em Adultos E Idosos De Comunidades Quilombolas Do Centro-Sul Do Piauí, Brasil. Estud Interdiscip sobre o Envelhec. 2014;19(2).

51. Oliveira EP, Souza MLA, Lima MDDA. Prevalence of metabolic syndrome in a semiarid rural area in Bahia. Arq Bras Endocrinol Metabol. 2006;50(3):456-65.

52. Haab RS, Benvegnú LA, Fischer EV. Prevalência de Síndrome Metabólica em uma área rural de Santa Rosa. Rev Bras Med Família e Comunidade. 2012;7(23):90-9.

53. Leitão MPC, Martins IS. Prevalência e fatores associados à síndrome metabólica em usuários de unidades básicas de saúde em São Paulo - sp. Rev Assoc Med Bras. 2012;58(1):60-9.

54. Campos C, Medeiros M, Ramos AT, Aparecida M, Cardoso A, Xavier IS, et al. Insulin Resistance and its Association with Metabolic Syndrome Components. Arq Bras Cardiol. 2011;97(5):380-9. 
medRxiv preprint doi: https://doi.org/10.1101/2021.06.03.21258301; this version posted June 4, 2021. The copyright holder for this preprint (which was not certified by peer review) is the author/funder, who has granted medRxiv a license to display the preprint in perpetuity.

55. Liu W, Cao Y, Dong L, Zhu Y, Wu Y, Lv Z, et al. cardiovascular disease in people with periodontitis ( Review ). Cochrane Database Syst Rev. 2019;(12).

56. Baeza M, Morales A, Cisterna C, Cavalla F, Jara G, Isamitt Y, et al. Effect of periodontal treatment in patients with periodontitis and diabetes: Systematic review and metaanalysis. J Appl Oral Sci. 2020;28:1-13.

57. Souza MDG de, Vilar L, Andrade CB de, Albuquerque R de O, Cordeiro LH de O, Campos JM, et al. Prevalência De Obesidade E Síndrome Metabólica Em Frequentadores De Um Parque. ABCD Arq Bras Cir Dig (São Paulo). 2015;28(suppl $1): 31-5$.

58. Renner S, Blutke A, Dobenecker B, Dhom G, Müller TD, Finan B, et al. Metabolic syndrome and extensive adipose tissue inflammation in morbidly obese Göttingen minipigs. Mol Metab. 2018;16(June):180-90.

59. Vettore MV, de Amorim Marques RA, Peres MA. Social inequalities and periodontal disease: Multilevel approach in SBBrasil 2010 survey. Rev Saude Publica. 2014;47(SUPPL.3):29-39.

60. Chalub LLF, Péret A de CA. Desempenho Do Índice Periodontal Comunitário (Cpi) Na Determinação Da Condição Periodontal: Enfoque No Exame Parcial. Arq Bras Odontol. 2010;6(3):155-62.

61. Adachi N, Kobayashi Y. One-year follow-up study on associations between dental caries, periodontitis, and metabolic syndrome. J Oral Sci. 2020;62(1):52-6.

62. Loesche WJ. Periodontal disease: link to cardiovascular disease. Compend Contin Educ Dent. 2000;21(6):463-70.

63. Almeida RF, Morado Pinho M, Lima C, Faria I, Santos P, Bordalo C. Associação entre doença periodontal e patologias sistémicas. Rev Port Clínica Geral. 2006;22:379-90. 